\title{
Retinal Blood Vessel Segmentation by Means of Scale-Space Analysis and Region Growing
}

\author{
M. Elena Martínez-Pérez ${ }^{1}$, Alun D. Hughes ${ }^{2}$, Alice V. Stanton ${ }^{2}$, \\ Simon A. Thom ${ }^{2}$, Anil A. Bharath ${ }^{1}$, and Kim H. Parker ${ }^{1}$ \\ 1 Department of Biological and Medical Systems \\ Imperial College of Science, Technology and Medicine \\ Prince Consort Road, London, SW7 2BY, UK \\ 2 Department of Clinical Pharmacology \\ Imperial College of Science, School of Medicine at St. Mary's \\ Paddington, London, W2 1NY, UK
}

\begin{abstract}
We present a method for retinal blood vessel segmentation based upon the scale-space analysis of the first and second derivative of the intensity image which gives information about its topology and overcomes the problem of variations in contrast inherent in these images. We use the local maxima over scales of the magnitude of the gradient and the maximum principal curvature as the two features used in a region growing procedure. In the first stage, the growth is constrained to regions of low gradient magnitude. In the final stage this constraint is relaxed to allow borders between regions to be defined. The algorithm is tested in both red-free and fluorescein retinal images.
\end{abstract}

\section{Introduction}

The eye is a window to the retinal vascular system which is uniquely accessible for the non-invasive, in vivo study of a complete vascular bed in humans. The detection and measurement of blood vessels can be used to quantify the severity of disease or as part of the process of automated diagnosis of disease. Retinal blood vessels can have measurable geometrical changes, in diameter, branching angles, lengths or tortuosity, as a result of a disease [4]. Thus a reliable method of vessel segmentation is needed for the early detection and characterisation of changes due to such diseases.

Different techniques are used to acquire images of retinal blood vessels. A relatively non-invasive technique, widely used clinically, is the retinal fundal photograph taken using a green filter. A more invasive technique is fluorescein angiography which involves an intravenous injection of dye which increases the contrast of the blood vessels against the background (Figure 3).

Previous studies have been carried out on the detection or enhancement of blood vessels in general and retinal blood vessels in particular [7]. Most of the work on segmentation of retinal images have been based in edge detectors or matched filters [1]. We have applied these two methods but because of the large regional variations in intensity inherent in these images and the very low contrast 
between vessels and the background, particularly in the red-free photographs, the results were disappointing. Techniques based on edge detectors lacked robustness in defining blood vessels without fragmentation and techniques based on matched filters were difficult to adapt to the variations of widths and orientation of blood vessels.

Multiscale analysis of the second derivative information was used to develop a vessel enhancement filter on X-ray images [3]. We present a method based on scale-space analysis from which we obtain retinal blood vessel width, size and orientation using two main geometrical features based upon the first and the second derivative of the intensity (edges and the maximum principal curvature) along the scale-space, that give information about the topology of the image. We then use a multiple pass region growing procedure which progressively segments the blood vessels using the feature information together with spatial information about the 8-neighbouring pixels.

\section{Method}

\subsection{Scale-Space Representation}

The idea behind scale-space representation is to separate out information at different scales. Any image can be embedded in one-parameter family of derived images $I(x, y ; s)$ obtained by convolving the original image $I(x, y)$ with a Gaussian kernel $G(x, y ; s)$ of variance $s^{2}$ :

$$
I(x, y ; s)=I(x, y) \otimes G(x, y ; s)
$$

where $s$ is a length scale factor. Figure 1(a) shows different scale-space representations of a portion of a red-free retinal image. Each scale slice is a version of the original after some amount of blurring, hence fine scale details disappear and images become more diffuse when scale parameter increases [5]. The use of Gaussian kernels to generate the scale-space information ensures that the objects in the images are invariant with respect to translation, rotation and size (scaling).

Under this framework, the derivative of an image $I(x, y)$ is defined as the linear convolution of the image with scale-normalised derivative of Gaussian kernels.

$$
\begin{array}{ll}
I_{x}=I(x, y) \otimes s G_{x} ; & I_{x x}=I(x, y) \otimes s^{2} G_{x x} \\
I_{y}=I(x, y) \otimes s G_{y} ; & I_{x y}=I(x, y) \otimes s^{2} G_{x y} \\
& I_{y y}=I(x, y) \otimes s^{2} G_{y y}
\end{array}
$$

where subscripts indicate partial derivatives and

$$
G(x, y ; s)=\frac{1}{2 \pi s^{2}} e^{-\frac{x^{2}+y^{2}}{2 s^{2}}}
$$




$$
s=2
$$
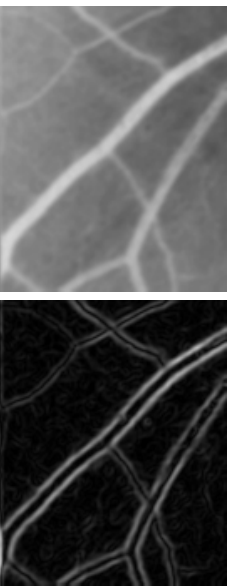

(c)
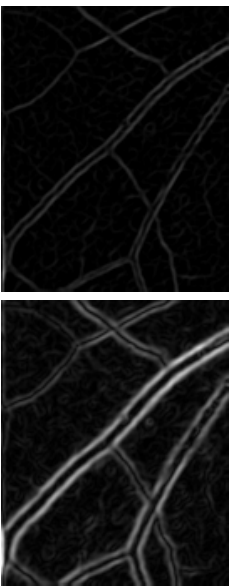

(d)

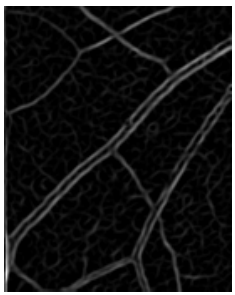

$s=8$
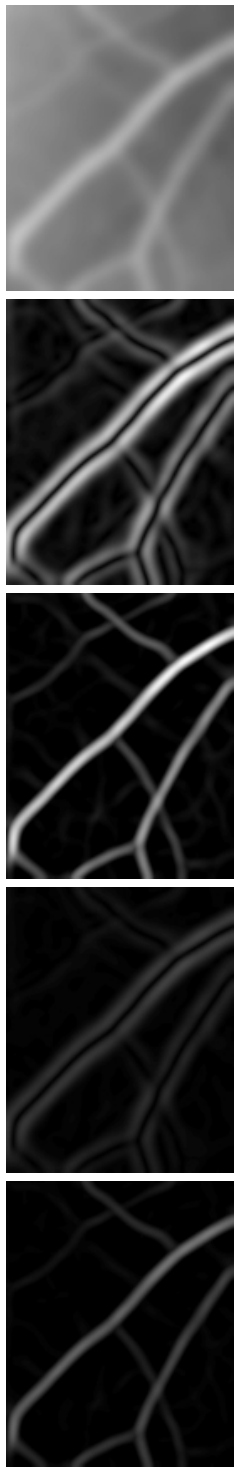

$s=14$
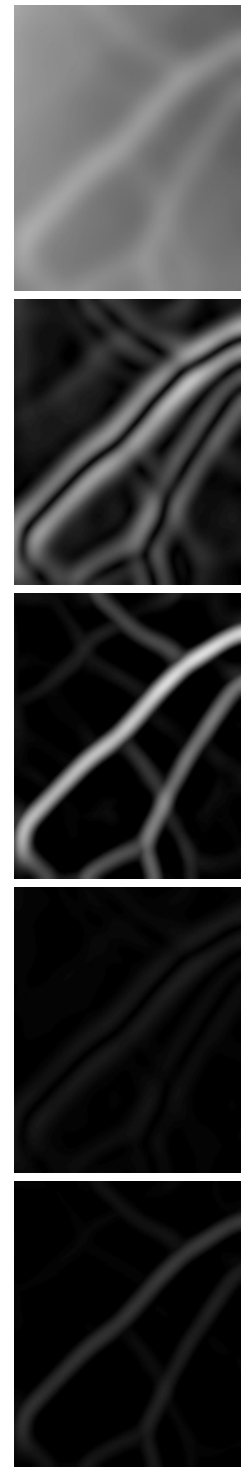

Maxima over scales
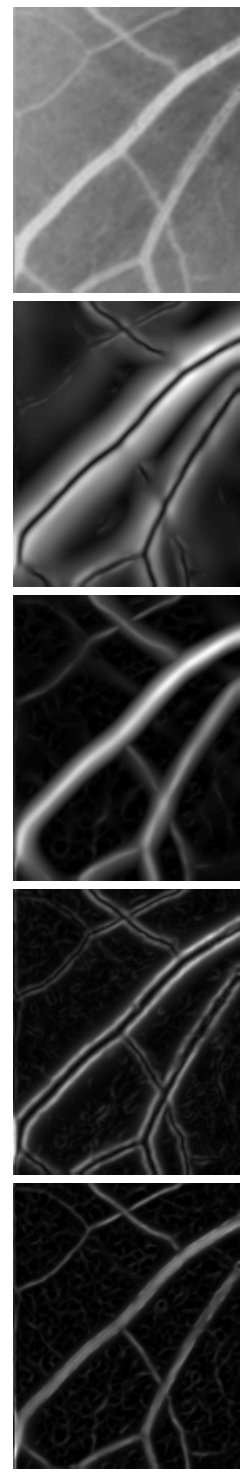

Fig. 1. Scale-space analysis for $s=2,8$ and 14 of a portion $(360 \times 290)$ of a red-free retinal image $(1400 \times 1200)$. (a) Original image, $I(s)$, (b) magnitude of the gradient, $|\nabla I(s)|$, (c) maximum principal curvature, $\left|\lambda_{2}(s)\right|$, (d) intensity scaled magnitude of the gradient, $|\nabla I(s)| / s$ and (e), intensity scaled maximum principal curvature, $\left|\lambda_{2}(s)\right| / s$. The last column in the first row is the original image and in the other rows is the maxima over scales. 


\subsection{Feature Extraction}

The measurements of feature strength that we used to characterise blood vessels are edges and maximum principal curvatures (ridges).

Edge strength. An edge point is weighted by the magnitude of the gradient of the image. The gradient is a vector function which represents the changes in intensity in the coordinate directions, and its magnitude is equal to the value of the slope, which is high at the edges and low at uniform regions:

$$
|\nabla I|=\sqrt{I_{x}^{2}+I_{y}^{2}}
$$

Ridge strength. A ridge point is a point for which the intensity image has a local maximum in the direction for which the gradient of the image undergoes the largest change (largest concavity) [2]. The second derivative information can be derived from the Hessian of the intensity image $I(x, y)$ :

$$
H=\left(\begin{array}{ll}
I_{x x} & I_{x y} \\
I_{y x} & I_{y y}
\end{array}\right)
$$

Since $I_{x y}=I_{y x}$ the Hessian matrix is symmetrical with real eigenvalues and orthogonal eigenvectors which are rotation invariant. The eigenvalues of the Hessian, $\lambda_{1}$ and $\lambda_{2}$, where we take $\left|\lambda_{1}\right| \leq\left|\lambda_{2}\right|$, measure convexity and concavity in the corresponding eigendirections. The maximum eigenvalue $\left(\left|\lambda_{2}\right|\right)$ will correspond to the maximum principal curvature. Thus, a pixel belonging to a vessel region will be weighted as a ridge pixel if $\left|\lambda_{2}\right| \gg 1$, for both red-free and fluorescein images (Figures 1(b) and (c)).

Intensity scaling. From last column of Figures 1(b) and (c), it is noticeable that the local maxima response is much higher for large blood vessels than for small ones. This might be expected since the vessels are approximately cylindrical so that the total amount of blood in the light path corresponding to each pixel is larger in large vessels. Thus, there will be more absorption of non-red light in the red-free images and increased fluorescence in fluorescein images in the larger vessels.

To account for this effect, we introduce an intensity scale factor which is related to the size of the blood vessels. Because the parameter $s$ is related to the width of the vessels, we normalise each feature with this factor over the scale-space and then keep the local maxima:

$$
\gamma=\max _{s}\left[\frac{|\nabla I(s)|}{s}\right] \quad ; \quad \kappa=\max _{s}\left[\frac{\left|\lambda_{2}(s)\right|}{s}\right]
$$

Figures 1(d) and (e) show these two new scale-space representations with the local maxima over scales in the last column.

\subsection{Using the Scale-Space Information}

We calculate the scale-space information in intervals $s_{\min } \leq s \leq s_{\max }$ where $s_{\min }$ and $s_{\max }$ are fixed according to the sizes of the smallest and largest vessels to be detected in the image. In this case we use $2 \leq s \leq 20$ with steps of 1 . 
The approach we use to extract information across the scales is to keep the local maxima over scales for both measurements of feature strength. These values, the local maxima of the intensity scaled gradient magnitude, $\gamma$, and the local maxima of the intensity scaled maximum principal curvature, $\kappa$, are then used as the two features to classify pixels in the image into two region classes, background and vessel, using a multiple pass region growing procedure.

\subsection{Region Growing}

The labelling algorithm is designed using information from the histograms of both features $h(\gamma)$ and $h(\kappa)$ and spatial information from the 8-neighbouring pixels. In the first stage the growing for both classes is restricted to regions with low gradients, allowing rapid growth of regions outside of the boundaries, and allowing vessels to grow where the values of $\kappa$ lie within a wide interval.

For $h(\gamma)$ only one class is used: low gradient, which is defined as $\gamma<$ $\mu_{g}+\sigma_{g}$ for the complete histogram (Figure $2(\mathrm{a})) . h(\kappa)$ is divided into two classes using the Otsu threshold algorithm [6], and their means and standard deviations are calculated: background, for $\kappa / \kappa_{\max } \in[0, t]$ with mean $\mu_{b}$ and variance $\sigma_{b}^{2}$; and vessel, for $\kappa / \kappa_{\max } \in(t, 1]$ with mean $\mu_{v}$ and variance $\sigma_{v}^{2}$, where $t$ is the threshold (Figure 2(b)).

The algorithm begins by planting seeds for each region: background seeds are pixels for which $\kappa \leq \mu_{b}$, whereas vessel seeds are defined as $\kappa \geq \mu_{v}$. Region growing is by an iterative process: An unlabelled pixel is classified as belonging to class $i$ if it fulfils a specific condition with initial parameters $a_{i}=1$. Growing is repeated until no more pixels are classified. The constraints are relaxed by incrementing the parameters $a_{i}$ by 0.5 and the growing is repeated.
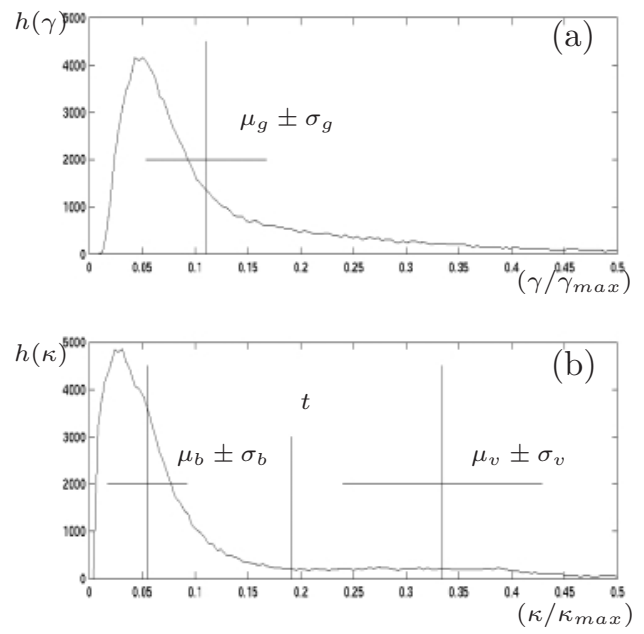

Fig. 2. Parameters used in the region growing algorithm. (a) Histogram of the local maxima of the edge strength, $\gamma$. One class: low gradient, $\gamma<\mu_{g}+\sigma_{g}$. (b) Histogram of the local maxima of the ridge strength, $\kappa$, where $t$ is the threshold. Two classes: background, $\kappa / \kappa_{\max } \in[0, t]$ and vessel, $\kappa / \kappa_{\text {max }} \in(t, 1]$.

For the first stage, the condition for class vessel is:

$$
\left(\mu_{v}-a_{v} \sigma_{v}\right) \leq \kappa A N D \quad \gamma \leq\left(\mu_{g}+a_{g} \sigma_{g}\right) A N D \quad N_{v} \geq 1
$$

whereas the condition for class background is:

$$
\kappa \leq\left(\mu_{b}+a_{b} \sigma_{b}\right) \text { AND } \gamma \leq \mu_{g} \text { AND } N_{b} \geq 1
$$


where $N_{i}$ is the number of neighbours already labelled as class $i$.

After alternating these two procedures until no further classifications are found, the final stage of the algorithm grows vessel and background classes simultaneously without the gradient restriction. Now the condition for class $i$ is:

$$
\left(\mu_{i}-a \sigma_{i}\right) \leq \kappa \leq\left(\mu_{i}+a \sigma_{i}\right) \quad A N D \quad N_{i} \geq 1
$$

and again the condition is relaxed by increasing the value of $a$ until all pixels are classified. With this final stage, borders between classes are defined. The complete procedure was applied to both red-free and a fluorescein images. Results are shown in Figure 3.

\section{Results and Conclusion}

From these results it can be seen that despite the much poorer contrast, the red-free image segmentation is nearly as good as that of the fluorescein image. We also find that the segmentation is relatively insensitive to the wide variations in intensity that are inherent in these images. Note in the fluorescein image, for example, the similar detail in the segmented image in the much brighter region just below the darker optic disk in the upper centre of the image.

It should be pointed out that although the idea of the intensity scale factor applied to the scale-space information is empirical, it can be justified by a simple model in which the intensity of the image is proportional to the amount of blood in the light path corresponding to that pixel. In any case, the method seems to work well in detecting retinal blood vessels over a large range of widths.

We have presented an algorithm which combines: 1) the scale-space representation that gives information about width, length and orientation of blood vessels, 2) two important geometrical properties of tube-like structures based on the first and second derivative information which give weights to pixels with a high probability of belonging to vessels, and 3) a multiple pass region growing procedure. The region growing algorithm is relatively fast because in the initial stage growth is restricted to regions with low gradients, allowing vessels to grow where the values of the maximum principal curvature lie within a wide interval. This allows rapid growth of regions outside the boundaries. In the final stage when the borders between classes are defined, the algorithm grows vessel and background classes simultaneously without the gradient restriction.

The appearance of the retinal blood vessels can be an important diagnostic indicator of various disorders of the eye and the body. From the segmented images, the morphology of retinal blood vessels can be measured in order to study changes resulting from disease: geometrical factors such as diameters, branching angles, lengths, tortuosity, etc. and network properties such as connectivity, branch ordering and, if appropriate, fractal properties. We are currently applying this segmentation algorithm to analyse these properties using clinical fundus photographs from normal subjects and hypertensive patients. 
(a)
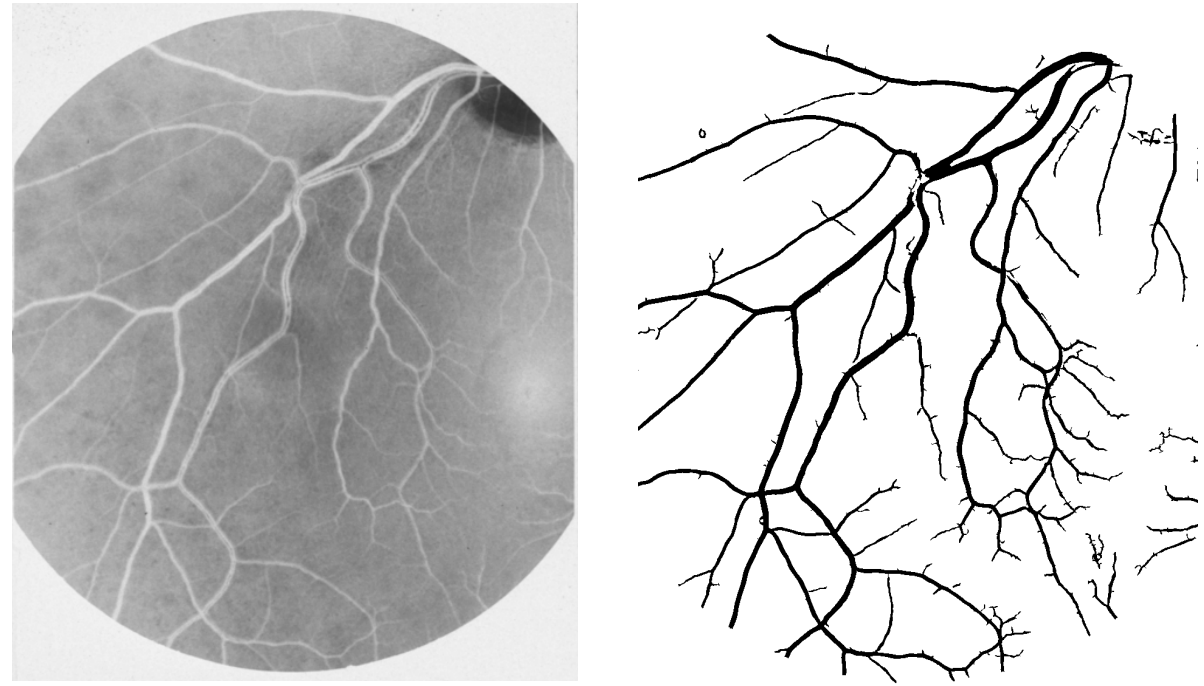

(b)
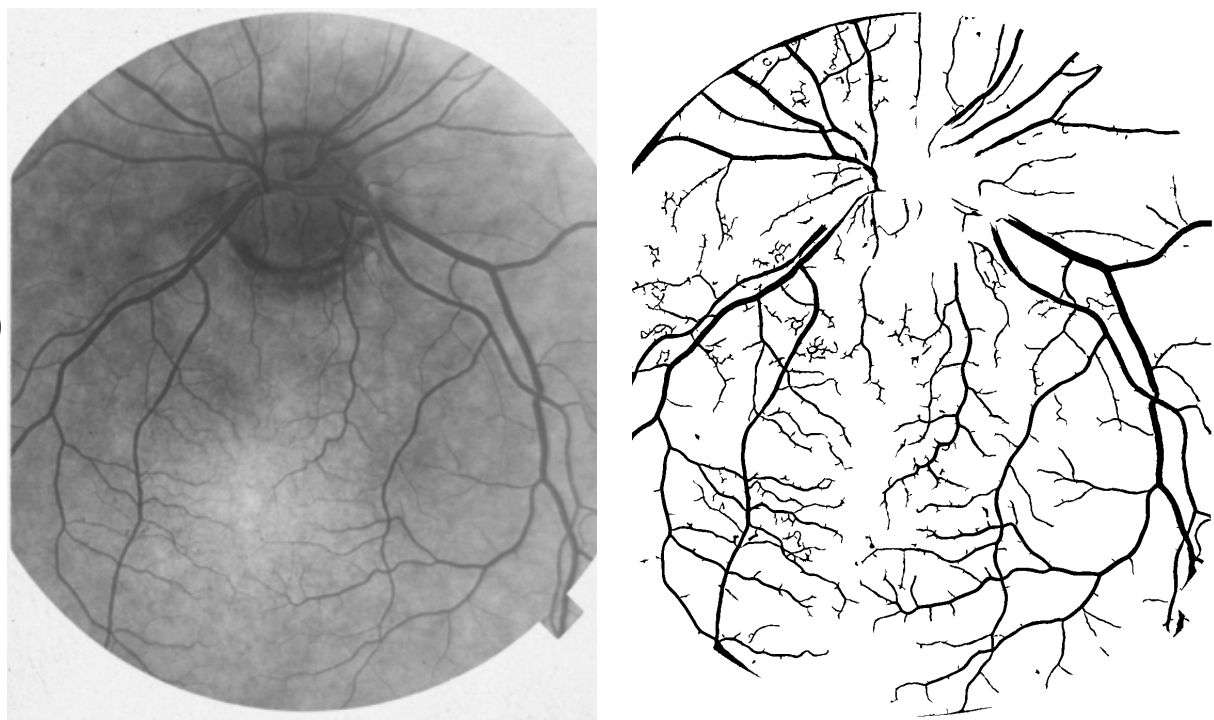

Fig. 3. Application of scale-space analysis and region growing segmentation to (a) red-free and (b) fluorescein fundal retinal images. The first column shows the original images. The second column shows the segmented vessels. Both images were photographed with the same resolution (size $1400 \times 1200$ pixels), the images correspond to the scanned negatives. 


\section{References}

1. S. Chaudhuri, S. Chatterjee, N Katz, M. Nelson, and M. Goldbaum. Detection of blood vessels in retinal images using two-dimensional matched filters. IEEE Trans. Med. Imag., 8:263-269, 1989. 90

2. D. Eberly. Ridges in Image and Data Analysis. Computational Imaging and Vision. Kluwer Academic Publishers, Netherlands, 1996. 93

3. A.F. Frangi, W.J. Niessen, K.L. Vincken, and M.A. Viergever. Multiscale vessel enhancement filtering. In W.M. Wells, A. Colchester, and S. Delp, editors, Medical Image Computing and Computer-Assisted Intervantion- MICCAI'98, number 1496 in Lecture Notes in Computer Science, pages 130-137, Berlin, 1998. Springer. 91

4. L.A. King, A.V. Stanton, P.S. Sever, S. Thom, and A.D. Hughes. Arteriolar lengthdiameter (l:d) ratio: A geometric parameter of the retinal vasculature diagnostic of hypertension. J. of Hum. Hypertens., 10:417-418, 1996. 90

5. T. Lindeberg and B.M. ter Haar Romeny. Linear scale-space. In B.M. ter Haar Romeny, editor, Geometry-Driven Diffusion in Computer Vision, Computational Imaging and Vision, pages 1-72. Kluwer Academic Publishers, Dordrecht, 1994. 91

6. N. Otsu. A threshold selection method from gray-level histograms. IEEE Trans. Syst., Man, Cybern., SMC-9:62-66, Jan 1979. 94

7. S.A. Thom, X. Gao, A.V. Stanton, J.N. Chapman, A. Bharath, and A.D. Hughes. A computer system for automated retinal microvascular architecture quantification. J. Hypertens., 16(S2):1421, 1998. 90 